\title{
Molecular cluster decay viewed as escape from a potential of mean force
}

\author{
Ian J. Ford and Sarah A. Harris \\ Department of Physics and Astronomy, University College London, \\ Gower Street, London WC1E 6BT, United Kingdom.
}

\begin{abstract}
We show that evaporation from a quasi-stable molecular cluster may be treated as a kinetic problem involving the stochastically driven escape of a molecule from a potential of mean force. We derive expressions for the decay rate, and a relationship between the depth of the potential and the change in system free energy upon loss of a molecule from the cluster. This establishes a connection between kinetic and thermodynamic treatments of evaporation, but also reveals differences in the prefactor in the rate expression. We perform constant energy molecular dynamics simulations of cluster dynamics to calculate potentials of mean force, friction coefficients and effective temperatures for use in the kinetic analysis, and to compare the results with the directly observed escape rates. We also use the simulations to estimate the escape rates by a probabilistic analysis. It is much more efficient to calculate the decay rate by the methods we have developed than it is to monitor escape directly, making these approaches potentially useful for the assessment of molecular cluster stability.
\end{abstract}

\section{INTRODUCTION}

Vapours are not simply collections of separated molecules or monomers: they also contain molecular clusters, growing and evaporating by molecular gain and loss. These ephemeral condensed structures play a central role in the nucleation of aerosols from metastable, or supersaturated vapours. The bulk condensed phase is thermodynamically more stable than the metastable vapour, but the transition can only proceed through the growth of molecular clusters, and so their stability is crucial. If a cluster manages to grow larger than a certain critical size, it stands a good chance of becoming a macroscopic droplet, but the dynamical route by which molecules cluster and form a condensed phase is rather complicated. Given a configuration of $N$ molecules (a specification of the positions and momenta of all the atoms) and a set of intermolecular forces, we need to know how many large (supercritical) molecular agglomerates are likely to be produced after a certain time. We need to compute this number for an arbitrary choice of initial condition consistent with the constraints applied to the system, such as average density and temperature. The need to consider all possible initial states requires a use of statistics: an ensemble average.

The full characterisation of the dynamics would require knowledge of the complete trajectory of all $N$ particles. This is huge amount of information, and the traditional simplification is to classify the system in terms of the populations of molecular clusters contained within it as time progresses. Rather than following the time evolution of $6 \mathrm{~N}$ positions and momenta, the dynamics are represented by the evolution of the cluster populations. Often it is sufficient to monitor the cluster populations up to a maximum cluster size of the order of 100, and the amount of information involved is then considerably less than a full dynamical description.

The dynamics of population evolution for an ensemble of trajectories, starting from all conceivable initial states, can be modelled using a simple set of rate equations proposed by Becker and Döring ${ }^{1}$. The evolution of $n_{i}(t)$, the mean population at time $t$ of clusters consisting of $i$ molecules, is described by

$$
\frac{d n_{i}}{d t}=\beta_{i-1} n_{i-1}-\gamma_{i} n_{i}-\beta_{i} n_{i}+\gamma_{i+1} n_{i+1}
$$

where $\beta_{i}$ is the mean rate at which monomers attach to cluster of size $i$, and $\gamma_{i}$ is the rate at which they detach from the same cluster. The terms on the right hand side in equation (1) represent gain of $i$-clusters from the growth of $(i-1)$-clusters, loss by the decay to $(i-1)$-clusters, loss by growth to $(i+1)$-clusters, and gain by decay of $(i+1)$-clusters. The growth rates $\beta_{i}$ are proportional to the monomer population $n_{1}$. The attachment of dimers and larger clusters is ignored. The Becker-Döring equations may be solved for a metastable vapour to give a steady state nucleation rate, which is related to the proportion of all initial molecular configurations that evolve to produce a large growing agglomerate in a certain time interval.

Let us not forget, though, that the nucleation phenomenon is an example of irreversible thermodynamics, the statistical physics of systems far from equilibrium, and rigorous methods do not exist to treat such systems mathematically ${ }^{2}$, though near-equilibrium approximations are available. The complicated real molecular dynamics are represented in the Becker-Döring treatment by the simple rate equations shown above. The growth and decay processes are assumed to proceed at rates that depend only on the gross properties of the system (temperature, etc) and not on the previous history of individual clusters, or indeed of populations of clusters. This is equivalent to saying that the transition processes are Markovian: there is a constant probability per unit time that a cluster will gain or lose a molecule. So it is important to note that the Becker-Döring equations are empirical equations constructed to solve an idealised problem: the validity of the approximations when applying them to real nucleating systems has not been established. Despite these simplifying assumptions, the Becker-Döring model (and its various extensions) is a very useful approach. The equations can be solved analytically, which is a great advantage. It is a true kinetic treatment of nucleation, requiring only knowledge of the mean rates of cluster growth and decay. 
The main alternative point of view for describing the nucleation process is based on thermodynamics, or more particularly the theory of free energy fluctuations. One identifies a transition state, again a molecular cluster, which is in unstable thermodynamic equilibrium with the metastable vapour ${ }^{3}$. According to the theory of free energy fluctuations, such a state is formed with probability proportional to $\exp \left(-\Delta W^{*} / k T\right)$, where $\Delta W^{*}$ is the reversible work of formation of the cluster. This approach is very useful, though it is often implemented using continuum thermodynamics ideas ${ }^{4}$, and applying these to small molecular agglomerates raises a number of questions and problems. The classical theory of nucleation may be derived in this way, by treating the transition state as though it were a macroscopic droplet. Microscopic calculations of cluster free energies are more acceptable, but more laborious.

One can establish a connection between the free energy fluctuation theory and the Becker-Döring treatment if the rate coefficients in the latter are expressed as differences in free energy between various cluster sizes ${ }^{5}$. Although there is some uncertainty in the mapping, this connection can be usefully exploited. The decay rate in a kinetic treatment of cluster population dynamics is difficult to calculate, and so it is useful to be able to relate it to a thermodynamic quantity and then to calculate this quantity through equilibrium statistical mechanics ${ }^{6-12}$.

There is also scope for calculating a decay rate using the near-equilibrium statistical mechanical techniques mentioned above, which employ a mix of equilibrium thermodynamic properties of clusters and the kinetics of change. This brings a notion of time into equilibrium thermodynamics which is otherwise absent, and which has to be added by ad-hoc arguments from the kinetic theory of gases. The methods are based on linearised non-equilibrium thermodynamics going back to Onsager ${ }^{13}$, and developed for this application by Reguera et $\mathrm{al}^{14}$ and by Schenter et al. ${ }^{15}$.

Implicit in any microscopic theory of nucleation, however, is the need for a clear definition of what is meant by a cluster. This is a subtle matter, and one which has received considerable attention ${ }^{6,7,15-26}$. Intuitively, a cluster should comprise a set of molecules located close to one another. The simplest definitions employed are indeed geometric, requiring the molecules to lie within a specified volume, or within a certain distance of one another. Selecting the arbitrary confining volume or maximum molecular separation is not necessarily a problem: these are essentially variational parameters, chosen to match the free energy of the system described by cluster populations to the true free energy of the system described by the $6 \mathrm{~N}$ degrees of freedom. However, with geometric definitions no attempt can be made to eliminate situations where component molecules are not energetically bound to the cluster. This has a consequence that the decay of a cluster defined in such a manner is then not Markovian. The rogue decays consist of situations where a molecule is unbound and simply passing by the other molecules. When it passes out of range, the 'cluster' would decay. However, the probability of cluster decay in these circumstances is not independent of time: it depends on when the passer-by first came within range of the other molecules. The required time independence of a Markovian decay rate is a characteristic of dynamics where molecules are bound for times much longer than the time taken for a molecule to cross the cluster at a typical velocity. Escape is stochastic, caused by the concentration of energy in one molecule by a random series of collisions.

We have recently developed a definition of a cluster involving energy rather than position ${ }^{24}$. It is widely recognised that an energetic rather than a positional criterion is an indicator of a quasi-bound structure ${ }^{19,23,27}$. We have added the essential feature that in order to escape from a cluster, a molecule needs not only to acquire positive energy, through thermal fluctuations, but must also be able to move away from the cluster, avoiding recapture. In order to check this second requirement, it is necessary to perform molecular dynamics to determine the future trajectory of the system. It is possible to implement such a scheme, and to determine mean decay rates as a function of cluster energy and size ${ }^{24}$. Similar studies of the molecular dynamics of condensation and escape have been performed by others, notably Schaaf et $a l^{26}$. We find that the decay rate is Markovian, so that clusters defined in our physically realistic scheme show the necessary features for use in the Becker-Döring equations.

This paper takes our ideas a stage further. Calculating mean decay rates by counting escaping molecules in molecular dynamics is quite time-consuming, and it would be valuable to be able to extract this information in some other way. This would allow our methodology to be extended to more complex systems that would be too computationally demanding to treat by direct simulation. Our strategy is to represent the decay as a stochastic process, described by a suitable mathematical scheme, and then to determine the parameters which enter that scheme by studying the cluster trajectory.

In this paper we also illustrate the connection between kinetic and thermodynamic treatments of nucleation. Kinetic theories of nucleation are based on models of the elementary rates of cluster growth and decay, while thermodynamic models rely on calculating the work of formation $\Delta W^{*}$ of an unstable critical cluster. In section II, we consider the Langevin dynamics of molecular escape from a cluster, and show that the rate of escape depends on the depth of the potential of mean force holding a particle in the cluster. The potential of mean force is also related to the steady state one-particle density profile. A statistical mechanical analysis is then used in section III to establish that the depth of this potential is related to a change in free energy associated with cluster decay. Using these results, we can show that the kinetic nucleation rate is proportional to $\exp \left(-\Delta W^{*} / k T\right)$, and hence that the Becker-Döring kinetic treatment is equivalent to the thermodynamic treatment, at least in certain circumstances. Furthermore, we can avoid the calculation of cluster free energies ${ }^{7-10,12}$ if we wish, and compute potentials of mean force from molecular dynamics simulations instead, and hence calculate decay rates. This could be a more convenient route to the determination of nucleation rates.

In section IV we illustrate these connections by estimating cluster decay rates in various ways. We calculate potentials of mean force, friction coefficients and particle density profiles, and hence a kinetic decay rate. We consider two versions of the 
rate prefactor: one involving the Langevin friction coefficient, and the other based on the principle of detailed balance. We go on to describe a further approach to the problem based on purely probabilistic arguments. The information needed for all these schemes can be extracted efficiently from the molecular dynamics simulations. We show that the estimated mean lifetimes closely match the lifetimes obtained by direct counting of molecular escapes. In section V we draw our conclusions and comment on the application of our methods to more complex situations.

\section{KINETIC THEORY OF CLUSTER DECAY}

We begin by taking the point of view that cluster decay corresponds fundamentally to the escape of a molecule from a three dimensional potential well created by the other molecules, driven by a random force. Nowakowski and Ruckenstein ${ }^{28,29}$ developed models of cluster decay starting from a similar assumption. However, they modelled the escape as a diffusive process along an energy coordinate, while we consider the motion of a molecule in real space.

We model the radial motion of an individual molecule, with respect to the cluster centre of mass, using a stochastic differential equation:

$$
m \ddot{r}=f(r)-m \gamma \dot{r}+\tilde{f}(r, t)
$$

where $r$ is the radial position, $m$ is the molecular mass. The right hand side of equation (2) is the stochastic force on the molecule, representing the interactions with the other molecules in the cluster. $f(r)$ is the mean (time- and velocity-averaged) force on the molecule at position $r$. The second and third terms on the right hand side of equation (2) introduce deviations from the mean force: the velocity dependence of this deviation is described using a dissipative term involving the friction coefficient $\gamma$ (not to be confused with the cluster decay rate $\gamma_{i}$ ). It represents the drag experienced by a molecule moving through a cloud of other molecules. The stochastic nature of the problem is represented by $\tilde{f}$, a velocity- and position-independent random force, with zero mean and correlation function $\left\langle\tilde{f}(r, t) \tilde{f}\left(r, t^{\prime}\right)\right\rangle=(2 \gamma k T / m) \delta\left(t-t^{\prime}\right)$, where $k$ is Boltzmann's constant and where $T$ has the characteristics of a temperature, as we shall see. Equation (2) clearly takes the form of Langevin's equation for noise-driven dissipative motion in a potential well.

It is a standard manipulation ${ }^{30}$ to convert the Langevin description, with large friction coefficient, into a Fokker-Planck, or Smoluchowski equation:

$$
\frac{\partial \mathcal{W}}{\partial t}=\frac{1}{m \gamma}\left(-\frac{\partial(f \mathcal{W})}{\partial r}+k T \frac{\partial^{2} \mathcal{W}}{\partial r^{2}}\right)
$$

which represents the evolution of $\mathcal{W}(r, t)$, the probability density that the molecule should lie at radial position $r$. The right hand side of the above equation may be written as $-\partial \mathcal{J} / \partial r$ where $\mathcal{I}$ is a radial probability current given by

$$
\mathcal{J}=\frac{1}{m \gamma}\left(f \mathcal{W}-k T \frac{\partial \mathcal{W}}{\partial r}\right)
$$

and so the steady state solution of equation (3) for the case when $\mathcal{I}=0$ is

$$
\mathcal{W}(r) \propto \exp (-\Phi(r) / k T)
$$

where $\Phi(r)$ is the potential of mean force, related to the mean force $f(r)$ through

$$
f=-\frac{d \Phi}{d r}
$$

We see now how the parameter $T$ in the random force plays the role of temperature, since equation (5) looks like a Boltzmann distribution. The $\mathcal{I}=0$ solution is not what we are seeking, however. The escape problem has a characteristic boundary condition $\mathcal{W}\left(r_{e}\right)=0$, where $r_{e}$ is the radius at which a particle escapes (is removed) from the system. We can implement this boundary condition by first writing the steady state current in the form

$$
\mathcal{J}=-\frac{k T}{m \gamma} \exp (-\Phi / k T) \frac{d}{d r}(\mathcal{W} \exp (\Phi / k T))
$$

The current $\mathcal{I}$ is found by integrating equation (7):

$$
\frac{k T}{m \gamma} \exp (\Phi(0) / k T) \mathcal{W}(0)=\int_{0}^{r_{e}} \mathcal{J} \exp (\Phi(r) / k T) d r
$$


which leads to

$$
\mathcal{J}=\mathcal{W}(0) \frac{k T}{m \gamma}\left(\int_{0}^{r_{e}} \exp ((\Phi(r)-\Phi(0)) / k T) d r\right)^{-1}
$$

This theory has been extensively applied ${ }^{31}$ to the case of particle escape over a barrier from a one-dimensional potential well, as illustrated in Figure 1(a). By expanding $\Phi(r)$ as $\Phi(r) \approx \Phi\left(r_{e}\right)-\frac{1}{2} m \omega_{e}^{2}\left(r-r_{e}\right)^{2}$ near the peak in the potential at a radius $r_{e}$, one can evaluate the integral in equation (9). Assuming further that the potential well is harmonic near $r=0$, so that $\Phi(r) \approx \Phi(0)+\frac{1}{2} m \omega^{2} r^{2}$, and deep compared with $k T$, one can also approximate $\mathcal{W}(r) \approx(2 / \pi)^{1 / 2} r_{0}^{-1} \exp \left(-r^{2} / 2 r_{0}^{2}\right)$, where the profile width $r_{0}$ is given by $r_{0}=(k T / m)^{1 / 2} \omega^{-1}$, where $\omega$ is the natural angular frequency of oscillation of a particle close to the bottom of the well. Recall that we are here considering a one dimensional problem so that $\mathcal{W}$ has dimensions of inverse length. Hence $\mathcal{W}(0) \approx(2 m / \pi k T)^{1 / 2} \omega$ and we obtain the escape rate ${ }^{32}$

$$
\mathcal{I}=\frac{2 \omega \omega_{e}}{\pi \gamma} \exp (-\Delta \Phi / k T)
$$

where $\Delta \Phi=\Phi\left(r_{e}\right)-\Phi(0)$ is the depth of the potential well.

In our case, however, we need to consider the escape of a molecule from a three dimensional potential of mean force into free space, as shown in Figure $1(\mathrm{~b}) . \mathcal{W}(r)$ is a probability per unit volume and the escape rate is $\gamma^{\mathrm{kin}}=4 \pi r_{e}^{2} \mathcal{g}$, where $r_{e}$ is the radius (apparently arbitrary at this point) at which escape is considered to take place. As before, we have $\mathcal{W}(0)=$ $\left(\int_{0}^{r_{e}} 4 \pi r^{2} \exp (-(\Phi(r)-\Phi(0)) / k T) d r\right)^{-1}$ and the escape rate is

$$
\gamma^{\text {kin }}=\frac{k T r_{e}^{2}}{m \gamma} \frac{\exp (-\Delta \Phi / k T)}{\left(\int_{0}^{r_{e}} \exp \left(\left(\Phi(r)-\Phi\left(r_{e}\right)\right) / k T\right) d r\right)\left(\int_{0}^{r_{e}} r^{2} \exp (-(\Phi(r)-\Phi(0)) / k T) d r\right)} .
$$

The value of $r_{e}$ seems arbitrary, but in fact it is related to the cluster definition; the mathematical scheme which determines whether a molecule may be classed as part of a cluster or not. We shall return to this point later. The principal feature of equation (11) is the exponential dependence on the depth $\Delta \Phi$ of the potential of mean force. The shape of the potential determines the integrals in the denominator. The time scale in the escape rate is provided by the friction coefficient. Therefore, if we can establish the potential of mean force, the effective temperature and the friction coefficient, by studying a molecular dynamics trajectory, for example, then we can use this Langevin analysis to determine the kinetic decay rate $\gamma^{\text {kin }}$.

\section{THERMODYNAMIC THEORY OF CLUSTER DECAY}

\section{A. Detailed balance in equilibrium}

We now turn our attention to relating the kinetic description of cluster decay just described to standard treatments of the problem starting from equilibrium thermodynamics. Such treatments involve free energy differences between clusters of various sizes. How does the cluster free energy relate to the potential of mean force, and what is the fundamental inverse timescale in the theory corresponding to the friction coefficient?

The thermodynamic, or equivalently statistical mechanical treatment of cluster decay is based on the following detailed balance condition in the population dynamics of clusters described by equation (1):

$$
\beta_{i-1} n_{i-1}^{e}=\gamma_{i} n_{i}^{e}
$$

where $n_{i}^{e}$ is the population of clusters of size $i$ in thermodynamic equilibrium with a vapour, which for convenience we take to be a saturated vapour. To a good approximation ${ }^{5}$, these populations are given by

$$
n_{i}^{e}=Z_{i} \exp \left(i \mu_{s} / k T\right)
$$

where $\mu_{s}$ is the chemical potential of the saturated vapour, and $Z_{i}$ is the cluster canonical partition function, given by

$$
Z_{i}=\frac{1}{i ! h^{3 i}} \int^{\prime} \prod_{k=1}^{i} d \mathbf{r}_{k} d \mathbf{p}_{k} \exp \left(-H_{i} / k T\right)
$$

where $h$ is Planck's constant, $\mathbf{r}_{k}$ and $\mathbf{p}_{k}$ are the position and momentum of particle $k$, and $H_{i}\left(\left\{\mathbf{r}_{k}, \mathbf{p}_{k}\right\}\right)$ is the cluster Hamiltonian, which takes the usual form $H_{i}=U\left(\left\{\mathbf{r}_{k}-\mathbf{r}_{l}\right\}\right)+\sum_{1}^{i} p_{k}^{2} / 2 m$ with $m$ representing the particle mass. The prime on the integral sign denotes the limitation of the phase space integration to molecular configurations satisfying a prescribed cluster definition. $Z_{i}$ is, 
of course, related to the cluster free energy $F_{i}$ through $Z_{i}=\exp \left(-F_{i} / k T\right)$. The growth rate $\beta_{i-1}$ is proportional to the population of monomers in the vapour, and so we can write $\beta_{i-1}=\beta_{i-1}^{\prime} n_{1}$. Hence, according to equation (13)

$$
\gamma_{i}=\beta_{i-1}^{\prime} \exp \left(-\left(F_{1}+F_{i-1}-F_{i}\right) / k T\right)=\beta_{i-1}^{\prime} \exp (-\Delta F / k T),
$$

where $\Delta F=F_{1}+F_{i-1}-F_{i}$ is the free energy change associated with monomer loss. It remains to evaluate the growth coefficient $\beta_{i-1}^{\prime}$, but this is not straightforward. In the absence of a better approach, the kinetic theory of collisions between a monomer and a spherical cluster is often used to provide the estimate $\beta_{i-1}^{\prime}=\left(R^{2} / V\right)(8 \pi k T / m)^{1 / 2}$, where $R$ is the somewhat ill-defined $(i-1)$-cluster radius, and $V$ is the system volume.

We now have two expressions, equations (11) and (15), for the cluster decay rate. These must be consistent with each other, at least in some circumstances. The principal similarity is the presence in each of an exponential of, respectively, the depth of the potential of mean force, and the change in free energy upon decay. We seek now to demonstrate that these quantities are related.

\section{B. The potential of mean force in statistical mechanics}

The strategy we shall follow is to evaluate the potential of mean force acting on a particle in the cluster using canonical statistical mechanics, and to see how it relates to cluster free energies.

Let us consider the mean radial force on a molecule at a distance $r_{1}$ from the centre of mass of a cluster of $i$ molecules. Without loss of generality, let us fix the origin of coordinates at the centre of mass of the system, and also set the total linear momentum to zero. The mean radial force is then given by the following phase space integral:

$$
f\left(\mathbf{r}_{1}\right)=\frac{1}{\xi\left(\mathbf{r}_{1}\right)} \int^{\prime} \prod_{k=2}^{i} d \mathbf{r}_{k} d \mathbf{p}_{k} d \mathbf{p}_{1} \delta\left(\sum_{k=1}^{i} \mathbf{r}_{k}\right) \delta\left(\sum_{k=1}^{i} \mathbf{p}_{k}\right)\left(-\frac{\partial H_{i}}{\partial r_{1}}\right) \exp \left(-H_{i} / k T\right),
$$

where the function

$$
\xi\left(\mathbf{r}_{1}\right)=\int^{\prime} \prod_{k=2}^{i} d \mathbf{r}_{k} d \mathbf{p}_{k} d \mathbf{p}_{1} \delta\left(\sum_{k=1}^{i} \mathbf{r}_{k}\right) \delta\left(\sum_{k=1}^{i} \mathbf{p}_{k}\right) \exp \left(-H_{i} / k T\right),
$$

when normalised is related to the equilibrium one-particle probability density:

$$
\rho(\mathbf{r})=\xi(\mathbf{r}) / \int^{\prime} \xi\left(\mathbf{r}_{1}\right) d \mathbf{r}_{1} .
$$

By symmetry, $\xi$ is a function of radius only. We proceed by considering its radial derivative:

$$
\begin{aligned}
\frac{d \xi}{d r_{1}} & =\frac{1}{k T} \int^{\prime} \prod_{k=2}^{i} d \mathbf{r}_{k} d \mathbf{p}_{k} d \mathbf{p}_{1} \delta\left(\sum_{k=1}^{i} \mathbf{r}_{k}\right) \delta\left(\sum_{k=1}^{i} \mathbf{p}_{k}\right)\left(-\frac{\partial H_{i}}{\partial r_{1}}\right) \exp \left(-H_{i} / k T\right) \\
& +\int^{\prime} \prod_{k=2}^{i} d \mathbf{r}_{k} d \mathbf{p}_{k} d \mathbf{p}_{1} \frac{\partial}{\partial r_{1}}\left(\delta\left(\sum_{k=1}^{i} \mathbf{r}_{k}\right)\right) \delta\left(\sum_{k=1}^{i} \mathbf{p}_{k}\right) \exp \left(-H_{i} / k T\right),
\end{aligned}
$$

assuming the integration limits do not depend on $r_{1}$. Fortunately, the second term on the right hand side of equation (19) can be simplified. We represent the derivative of the delta function as the limit of $\left(\delta\left(\sum_{2}^{i} \mathbf{r}_{k}+\left(r_{1}+\varepsilon\right) \hat{\mathbf{r}}_{1}\right)-\delta\left(\sum_{2}^{i} \mathbf{r}_{k}+\left(r_{1}-\varepsilon\right) \hat{\mathbf{r}}_{1}\right)\right) / 2 \varepsilon$ as $\varepsilon \rightarrow 0$, where $\hat{\mathbf{r}}_{1}$ is a unit vector in the direction of $\mathbf{r}_{1}$. Consider the integral

$$
I=\frac{1}{2 \varepsilon} \int^{\prime} \prod_{k=2}^{i} d \mathbf{r}_{k} d \mathbf{p}_{k} d \mathbf{p}_{1} \delta\left(\sum_{k=2}^{i} \mathbf{r}_{k}+\left(r_{1}+\varepsilon\right) \hat{\mathbf{r}}_{1}\right) \delta\left(\sum_{k=1}^{i} \mathbf{p}_{k}\right) \exp \left(-H_{i} / k T\right),
$$

and make the transformation $\mathbf{r}_{k} \rightarrow \mathbf{r}_{k}-\varepsilon \mathbf{r}_{1} /(i-1)$ for $k=2, i$. If $H_{i}$ is a function of spatial differences $\left(\mathbf{r}_{k}-\mathbf{r}_{l}\right)$ this term becomes

$$
I=\frac{1}{2 \varepsilon} \int^{\prime} \prod_{k=2}^{i} d \mathbf{r}_{k} d \mathbf{p}_{k} d \mathbf{p}_{1} \delta\left(\sum_{k=1}^{i} \mathbf{r}_{k}\right) \delta\left(\sum_{k=1}^{i} \mathbf{p}_{k}\right) \exp \left(-H_{i}\left(\mathbf{r}_{1}+\varepsilon \mathbf{r}_{1} /(i-1)\right) / k T\right),
$$

which may be expanded as

$$
I=\frac{1}{2 \varepsilon} \int^{\prime} \prod_{k=2}^{i} d \mathbf{r}_{k} d \mathbf{p}_{k} d \mathbf{p}_{1} \delta\left(\sum_{k=1}^{i} \mathbf{r}_{k}\right) \delta\left(\sum_{k=1}^{i} \mathbf{p}_{k}\right) \exp \left(-H_{i}\left(\mathbf{r}_{1}\right) / k T\right)\left(1-\frac{\varepsilon}{(i-1) k T} \frac{\partial H_{i}}{\partial r_{1}}\right)
$$


Hence

$$
\frac{d \xi}{d r_{1}}=\frac{1}{k T}\left(1+\frac{1}{i-1}\right) \int^{\prime} \prod_{k=2}^{i} d \mathbf{r}_{k} d \mathbf{p}_{k} d \mathbf{p}_{1} \delta\left(\sum_{k=1}^{i} \mathbf{r}_{k}\right) \delta\left(\sum_{k=1}^{i} \mathbf{p}_{k}\right)\left(-\frac{\partial H_{i}}{\partial r_{1}}\right) \exp \left(-H_{i} / k T\right)
$$

or more simply

$$
\frac{d \xi}{d r_{1}}=\frac{1}{k T}\left(\frac{i}{i-1}\right) f\left(r_{1}\right) \xi\left(r_{1}\right)
$$

which can be integrated to give

$$
\xi\left(r_{1}\right)=\xi(0) \exp \left(\frac{-i\left(\Phi\left(r_{1}\right)-\Phi(0)\right)}{(i-1) k T}\right)
$$

thus establishing through equation (18) a connection between the potential of mean force $\Phi$ and the equilibrium one-particle density profile:

$$
\rho\left(r_{1}\right) \propto \exp \left(-\frac{i \Phi\left(r_{1}\right)}{(i-1) k T}\right)
$$

This is the analogue of the particle probability density $\mathcal{W}(r)$ in the Langevin problem, which is related to the potential of mean force according to equation (5). In equation (26) we see an additional factor of $i /(i-1)$. It appears because the mean force on a particle in the cluster is created by the remaining $i-1$ particles with the added constraint that the centre of mass of the entire system lies at the origin. If the first delta function in equation (16) had not included $\mathbf{r}_{1}$ in the sum, then the factor $i /(i-1)$ in equation (26) would not have arisen. Thus the fixed centre of mass constraint is responsible for the difference between equations (5) and (26). As a check, consider a two particle system with interaction potential $\phi(r)$. When the radial displacement of particle 1 from the centre of mass is $r_{1}$, the mean force is $f\left(r_{1}\right)=-\phi^{\prime}\left(2 r_{1}\right)$. The potential of mean force is $\Phi\left(r_{1}\right)=-\int{ }^{r_{1}} f(r) d r=\int{ }^{r_{1}} \phi^{\prime}(2 r) d r=(1 / 2) \int^{2 r_{1}} \phi^{\prime}(y) d y=\phi\left(2 r_{1}\right) / 2$. Hence $\rho\left(r_{1}\right) \propto \exp \left(-\phi\left(2 r_{1}\right) / k T\right)$ in agreement with elementary expectations.

Now let us establish a connection between $\xi$ and a cluster partition function. Let us consider

$$
\frac{1}{h^{3}} \int Z_{i-1} \exp \left(-p_{1}^{2} / 2 m k T\right) d \mathbf{p}_{1}=\frac{1}{h^{3}} \frac{1}{(i-1) ! h^{3(i-1)}} \int^{\prime} \prod_{k=2}^{i} d \mathbf{r}_{k} d \mathbf{p}_{k} d \mathbf{p}_{1} \exp \left(-\left(H_{i-1}+p_{1}^{2} / 2 m\right) / k T\right),
$$

and then insert unit integrals $\int \delta\left(\sum_{1}^{i} \mathbf{r}_{k}-\mathbf{R}\right) d \mathbf{R}$ and $\int \delta\left(\sum_{1}^{i} \mathbf{p}_{k}-\mathbf{P}\right) d \mathbf{P}$ into the right hand side. Particle 1 introduced here is assumed to lie far away from the other particles. Next make a transformation of coordinates $\left\{\mathbf{r}_{k} \rightarrow \mathbf{r}_{k}-\mathbf{R} / i, \mathbf{R} \rightarrow \mathbf{R}\right\}$ for $k=1, i$, and $\left\{\mathbf{p}_{k} \rightarrow \mathbf{p}_{k}-\mathbf{P} / i, \mathbf{P} \rightarrow \mathbf{P}\right\}$ for $k=1, i$, for which the Jacobean is unity, giving

$$
\frac{Z_{i-1}}{\lambda^{3}}=\frac{1}{(i-1) ! h^{3 i}} \int^{\prime} \prod_{k=2}^{i} d \mathbf{r}_{k} d \mathbf{p}_{k} d \mathbf{p}_{1} \delta\left(\sum_{k=1}^{i} \mathbf{r}_{k}\right) d \mathbf{R} \delta\left(\sum_{k=1}^{i} \mathbf{p}_{k}\right) d \mathbf{P} \exp \left(-\left(H_{i-1}+p_{1}^{2} / 2 m+P^{2} / 2 m i^{2}\right) / k T\right)
$$

where $\lambda$ is the thermal de Broglie wavelength $\lambda=h /(2 \pi m k T)^{1 / 2}$. The particle positions $\mathbf{r}_{1}$ are measured with respect to the centre of mass $\mathbf{R} / i$, and the momenta with respect to the total momentum $\mathbf{P}$. Now, if particle 1 is very distant from the remaining particles, $H_{i-1}+p_{1}^{2} / 2 m \approx H_{i}$. Performing the integrals over $\mathbf{R}$ and $\mathbf{P}$ then gives

$$
\frac{Z_{i-1}}{\lambda^{3}}=\frac{V}{(i-1) ! h^{3(i-1)}} \frac{i^{3}}{\lambda^{3}} \int^{\prime} \prod_{k=2}^{i} d \mathbf{r}_{k} d \mathbf{p}_{k} d \mathbf{p}_{1} \delta\left(\sum_{k=1}^{i} \mathbf{r}_{k}\right) \delta\left(\sum_{k=1}^{i} \mathbf{p}_{k}\right) \exp \left(-H_{i}\left(r_{1} \rightarrow \infty\right) / k T\right),
$$

where $V$ is the system volume, or equivalently

$$
Z_{i-1}=\frac{V i^{3}}{(i-1) ! h^{3(i-1)}} \xi(\infty)
$$

Therefore we have established the connection

$$
\xi(\infty)=\frac{h^{3(i-1)}(i-1) !}{V i^{3}} \exp \left(-F_{i-1} / k T\right)
$$


Similarly, $\xi(0)$ is related to $F_{i}$. Equation (14) may be written, using the same insertions and transformations:

$$
Z_{i}=\frac{1}{i ! h^{3 i}} \int^{\prime} \prod_{k=1}^{i} d \mathbf{r}_{k} d \mathbf{p}_{k} \delta\left(\sum_{k=1}^{i} \mathbf{r}_{k}\right) d \mathbf{R} \delta\left(\sum_{k=1}^{i} \mathbf{p}_{k}\right) d \mathbf{P} \exp \left(-\left(H_{i}+P^{2} / 2 m i^{2}\right) / k T\right)
$$

which yields

$$
Z_{i}=\frac{1}{i ! h^{3 i}} \frac{h^{3} V i^{3}}{\lambda^{3}} \int^{\prime} \xi\left(\mathbf{r}_{1}\right) d \mathbf{r}_{1}
$$

so that $\int^{\prime} \xi\left(\mathbf{r}_{1}\right) d \mathbf{r}_{1}=\left(h^{3(i-1)} i ! \lambda^{3} / V i^{3}\right) \exp \left(-F_{i} / k T\right)$. However, $\int^{\prime} \xi\left(\mathbf{r}_{1}\right) d \mathbf{r}_{1}=\xi(0) / \rho(0)$ from equation (18), so

$$
\xi(0)=\rho(0) \frac{h^{3(i-1)} i ! \lambda^{3}}{V i^{3}} \exp \left(-F_{i} / k T\right) .
$$

Hence from equations (25), (31) and (34) we can establish our prime result

$$
\frac{1}{\lambda^{3}} \exp \left(-\left(F_{i-1}-F_{i}\right) / k T\right)=i \rho(0) \exp \left(\frac{-i(\Phi(\infty)-\Phi(0))}{(i-1) k T}\right)
$$

or equivalently

$$
F_{i}-F_{i-1}=k T \ln \left(i \rho_{i}(0) \lambda^{3}\right)-\frac{i}{i-1} \Delta \Phi_{i}
$$

where $\Delta \Phi_{i}=\Phi(\infty)-\Phi(0)$. The subscript $i$ on $\Delta \Phi_{i}$ indicates that the depth of the potential of mean force depends on cluster size, and similarly the one-particle density at the centre of mass is $i$-dependent, and hence the need for a subscript on $\rho(0)$.

The expression for the free energy change in equation (36) makes perfect physical sense, particularly if we rewrite it in the form

$$
\Delta F=F_{1}+F_{i-1}-F_{i}=\frac{i}{i-1} \Delta \Phi_{i}-k T \ln \left(i \rho_{i}(0) V\right)
$$

with $F_{1}=-k T \ln \left(V / \lambda^{3}\right)$. The left hand side is the change in free energy upon evaporation. The first term on the right hand side may be written as a sum of two terms $\left(1+\frac{1}{i-1}\right) \Delta \Phi_{i}$. The first term $\Delta \Phi_{i}$ is the reversible work done on a molecule by external forces when it is slowly dragged out of an $i$-cluster, but work is also done on the remaining molecules in order to keep the total centre of mass stationary. The total force on the remaining molecules is equal and opposite to that on the single molecule, but the distance their centre of mass moves is $i-1$ times smaller. The free energy change will therefore include this reversible work. This accounts for the second contribution to the sum. Furthermore, there is entropic change in free energy upon first identifying and holding one of the component molecules of the cluster stationary at the centre of mass, and then releasing it from the position outside the cluster to which it has been pulled. Considering the cluster for the moment to be a bag of volume $v(i)$, the first change is roughly $k T \ln (v(i) / i)$ (the factor of $1 / i$ to account for the choice in molecule) and the second is $-k T \ln V$. We note that $v(i) \sim 1 / \rho(0)$ and therefore recover the last term on the right hand side of equation (37).

Equation (35) is an exact relation between the depth of the potential of mean force confining a molecule to a cluster, and the difference in cluster free energy before and after the loss of that molecule. It suggests a method for calculating differences in free energy between $i$ - and $(i-1)$-clusters by evaluating the depth of the potential of mean force confining particles to an $i$-cluster, together with the particle probability density at the centre of mass. It is an alternative to methods such as umbrella sampling, which has been applied to this very problem ${ }^{9,12}$.

We now combine equation (37) with the detailed balance expression (15) to get the $i$-cluster decay rate:

$$
\gamma_{i}^{\text {therm }}=\left[\left(\frac{8 \pi k T}{m}\right)^{1 / 2} R^{2}\right] i \rho(0) \exp \left(\frac{-i \Delta \Phi_{i}}{(i-1) k T}\right) .
$$

This result is based on the assumption that the decay rate in a situation of detailed balance would apply for cases when the system is out of equilibrium. The prefactor in square brackets is an approximation based on kinetic theory for the collision rate of a monomer onto a spherical cluster of radius $R$.

Equation (38) should be compared with equation (11), the result from Langevin kinetics. First, though, we need to multiply the potential in equation (11) by a factor $i /(i-1)$, to take account of the condition of fixed centre of mass, and we must also 
multiply $\gamma^{\text {kin }}$ by $i$ since any one of the $i$ confined particles might escape. We should also add suffices $i$ to the well depth and the escape rate. We arrive at

$$
\gamma_{i}^{\mathrm{kin}}=\frac{i k T r_{e}^{2}}{m \gamma} \frac{\exp \left(-i \Delta \Phi_{i} /(i-1) k T\right)}{\left(\int_{0}^{r_{e}} \exp \left(i\left(\Phi(r)-\Phi\left(r_{e}\right)\right) /(i-1) k T\right) d r\right)\left(\int_{0}^{r_{e}} r^{2} \exp (-i(\Phi(r)-\Phi(0)) /(i-1) k T) d r\right)} .
$$

The principal difference between equations (38) and (39) then lies in the prefactors multiplying the exponential terms. This should not be a surprise since the Langevin equation approach, and the friction coefficient which appears in equation (??), is a phenomenological representation of the molecular dynamics, and as we have just noted, the prefactor in the thermodynamic escape rate is only approximate. The radii $r_{e}$ and $R$ in each expression ought to take approximately the same value, but they might differ. The reliability of the two prefactors may be judged by comparison with real escape rates, which we shall address in the next section.

First, though, there are some interesting further connections to draw between the potential of mean force and cluster properties. In the $i \rightarrow \infty$ limit, $F_{i}-F_{i-1}$ is equal to the chemical potential of the condensate and hence the chemical potential $\mu_{s}=k T \ln \left(p_{s} \lambda^{3} / k T\right)$ of a saturated vapour, where $p_{s}$ is the saturated vapour pressure. If we employ equation (36), and use $i \rho_{i}(0) \approx \rho_{l}$ in the limit of large $i$, where $\rho_{l}$ is the density of the condensate, then

$$
\Delta \Phi_{\infty}=-k T \ln \left(\frac{p_{s}}{\rho_{l} k T}\right) .
$$

This is similar to the Clausius-Clapeyron equation, showing that $\Delta \Phi_{\infty}$ is related to the molecular latent heat of evaporation.

Another useful procedure is to construct the equilibrium population $n_{i}^{e}$ of an $i$-cluster by repeated use of equation (36). We write

$$
F_{i}-F_{i-1}-\mu_{s}=\Delta \Phi_{\infty}-\frac{i}{i-1} \Delta \Phi_{i}+k T \ln \left(\frac{i \rho_{i}(0)}{\rho_{l}}\right)
$$

so that through equation (13),

$$
n_{i}^{e}=\exp \left(-\left(F_{i}-i \mu_{s}\right) / k T\right)=n_{2}^{e} \prod_{j=3}^{i} \frac{\rho_{l}}{j \rho_{j}(0)} \exp \left(-\frac{1}{k T} \sum_{j=3}^{i}\left(\Delta \Phi_{\infty}-\frac{j}{j-1} \Delta \Phi_{j}\right)\right) .
$$

The reference population chosen here is that of the dimer rather than the monomer since $\rho_{2}(0)$ is zero. However, the dimer population can be related to the monomer population through $n_{2}^{e} \approx-B_{2} V \rho_{v}^{2}$, where $B_{2}$ is the second virial coefficient of the vapour, defined through the equation $p=k T\left(\rho_{v}+B_{2} \rho_{v}^{2}\right)$, with $p$ and $\rho_{v}$ the vapour pressure and density respectively. Equation (42) is therefore an expression for the equilibrium population of $i$-clusters given in terms of quantities readily determined from molecular dynamics studies of clusters: densities at the centre of mass and depths of potentials of mean force. We do not need to calculate free energies explicitly. We intend to explore equation (42) in future work.

\section{CALCULATING THE ESCAPE RATE FROM MD SIMULATION}

\section{A. Cluster definition and molecular simulation}

We have previously described microcanonical molecular dynamics (MD) simulations of clusters of argon atoms ${ }^{24}$. These were performed both to implement new ideas for a realistic cluster definition and to compute cluster lifetimes for a variety of sizes and energies. Our aim is now to calculate the lifetime of these clusters indirectly by studying the simulation trajectory and evaluating the cluster properties needed in the theoretical formulae described in the previous section. We shall also develop here a third, simplified procedure for estimating the lifetime, using a probabilistic approach. These studies will demonstrate that the complex dynamical behaviour of molecular clusters can be described in terms of a simple model, parametrised through detailed MD simulations.

Any scheme to estimate the lifetime must implement a cluster definition. We consider that a particle becomes unbound when its kinetic energy $K$ becomes greater than the modulus of its potential energy $U$ (i.e. total energy is positive), but only if the dynamics subsequently carry the particle far away from the cluster, avoiding recapture. Not all particles that acquire positive energy necessarily escape. This is illustrated in Figure 2, which shows the total energy $K+U$ of a particle near the edge of a cluster as a function of time. Only one of the three positive energy excursions (indicated by arrows) lead to decay, giving a recapture probability $R_{c}=2 / 3$ in this example. From counting the proportion of positive energy excursions that lead to cluster decay in extensive MD simulations, the success factor $1-R_{c}$ was found to be about 0.12 for clusters of about 50 atoms. Only about one in eight positive energy excursions leads to escape. A higher proportion of particles escape when the clusters are smaller and the surface curvature is greater, as would be expected. 


\section{B. Potential of mean force approach}

\section{Langevin-derived prefactor}

We can use the MD simulations to calculate the potential of mean force, friction coefficient and temperature for use in the theoretical expressions for the decay rate derived in previous sections. The potential of mean force $\Phi(r)$ is found by averaging the force on a particle when at a radius $r$ from the cluster centre of mass. The temperature $T$ of the cluster is obtained by numerically fitting a Maxwell-Boltzmann distribution to the particle velocity distributions obtained from each trajectory.

The friction coefficient is found by mapping the actual molecular dynamics onto the Langevin dynamics. The radial component of the apparent acceleration of a particle $\mathbf{a}=(\mathbf{v}(t+\delta t / 2)-\mathbf{v}(t-\delta t / 2)) / \delta t$ for a given time interval $\delta t$ along the MD trajectory will not in general equal the radial component of the actual force on the particle at time $t$ divided by the mass, due to the finite value of $\delta t$. We ascribe the discrepancy to the sum of the friction force and the random force in the Langevin equation (2). It is clear then what to do: we average the mean discrepancy force, and plot it against particle velocity to extract the friction force element.

In Figure 3 we give such a plot for an example dataset with $\delta t=0.4 \mathrm{ps,} \mathrm{demonstrating} \mathrm{the} \mathrm{linear} \mathrm{correlation} \mathrm{between} \mathrm{friction}$ force and velocity. The slope of the dotted line fit to this behaviour is our estimate for $\gamma$. For different values of $\delta t$ a similar correlation is found, and the slope of the linear fit is shown against $\delta t$ in Figure 4 . For $\delta t \rightarrow 0$ molecular dynamics and not Langevin dynamics then holds so there is no discrepancy force and the apparent $\gamma$ goes to zero. For large $\delta t$, the correlation between force discrepancy and velocity is lost in noise from the random force contribution. There is a regime between these limits where the apparent friction coefficient is about $2.5 \mathrm{ps}^{-1}$, suggesting that Langevin dynamics is an adequate description for timescales $\delta t$ in the region of $0.4 \mathrm{ps}$.

A typical profile of potential of mean force $\Phi(r)$ for a cluster of $50 \operatorname{argon}$ atoms at $-2.98 \mathrm{~kJ} / \mathrm{mol}$ per particle (or about $T \approx 50.6$ $\mathrm{K}^{24}$ ) is shown in Figure 5. The profile for this size is reasonably flat at the cluster centre and then rises to a plateau. The oneparticle density profile given by equation (26) is therefore approximately uniform out to a radius of about $6 \AA$, and then falls to zero, as shown in Figure 6. This density profile is reminiscent of the density profiles calculated in density functional treatments of cluster structure ${ }^{33}$.

Now we can calculate the kinetic decay rate using equation (11). The results are shown in Figure 7 . We employ escape radii $r_{e}$ of $11,12,14.5$ and $17 \AA$ for $i=10,25,50$ and 100 respectively. These are estimates of the radii at which the potential of mean force first reaches zero for each cluster size, averaged over cluster energy. The condition for particle escape is therefore that the particle is able to reach the top of the free energy barrier, though this is a very rough treatment of a complicated problem. The idea is illustrated in Figure 5 for the case of the $i=50$ cluster at $-2.98 \mathrm{~kJ} / \mathrm{mol}$ per particle. The escape radius $r_{e}$ is in the region of the $14.5 \AA$ quoted. Plots for different energies show slightly different escape positions, and rather than complicate the model by using an energy dependent value of $r_{e}$, we choose a value dependent only on cluster size. Also note that it is rather delicate to determine the position of the 'lip' of the potential well when the data are noisy. The uncertainties are less crucial than any uncertainty in the depth of the well, however.

An energy-independent escape radius is equivalent to imposing a cluster definition that requires particles to lie within a given distance of the centre of mass, irrespective of energy. We have previously demonstrated that a geometric cluster definition with an energy-independent Stillinger radius cannot account for the observed cluster stabilities ${ }^{24}$. Therefore, deviations are therefore to be expected.

We can simplify the model so that only the depth of the well and not its shape enters the theoretical expression. We can parameterise of the model to fit to the data. If we assume the potential of mean force is a square well with depth $\Delta \Phi_{i}$ and radius $R_{s}$, then equation (39) reduces to

$$
\gamma_{i}^{\mathrm{kin}}=\frac{3 i r_{e} k T}{m \gamma R_{s}^{3}\left(1-\left(R_{s} / r_{e}\right)\right)} \exp \left(-i \Delta \Phi_{i} /(i-1) k T\right),
$$

if $\Delta \Phi_{i} \gg k T$. We can estimate the range of the square well $R_{s}$ using the one-particle profile $\rho(r)$ and the escape radius takes a position about one Lennard-Jones $\sigma(\sim 3.4 \AA)$ beyond this. Reasonable choices of the representative radii for the various cluster sizes can produce lifetimes in as good agreement with the directly observed data as the lifetimes given in Figure 7. Working with equation (43) has some advantages over equation (11) since the required input is easier to determine.

\section{Detailed balance-derived prefactor}

An alternative approach to estimating the decay rate is to employ equation (38), based on detailed balance and thermodynamics. This time we need to estimate the radius $R$ of a shell defining the capture cross-section of the cluster. Symmetry would suggest that we should estimate $R$ by determining the radius at which the particle escape is most likely. This radius is obtained for each cluster size using the probabilistic method described in the following section. This gives capture radii $R$ of $8,10,11$ and $12 \AA$ for $i=10,25,50$ and 100 respectively, as ilustrated for $i=50$ in Figure 9(e). 
We need estimates of the one-particle density at the origin $\rho(0)$ in order to use equation (38). For example, Figure 6 suggests a value of $5.0 \times 10^{-4} \AA^{-3}$ for the $i=50$ cluster. Similar analyses of data can provide $\rho(0)$ for all cluster sizes and energies in our simulations. We can then calculate the mean lifetimes shown in Figure 8. Once again, agreement with the directly observed mean lifetimes is reasonable, bearing in mind the estimation of $R$. Choosing $R$ on a different basis might improve the fit. The error in the predicted values is energy dependent, which is consistent with our previous conclusions that a geometric cluster definition cannot reproduce the correct energy dependence of decay rates.

We note that the prefactors in equations (38) and (43) have similar structure. If $\gamma^{-1}$ is a relaxation time, which we estimate to be a typical molecular separation $R / i^{1 / 3}$ divided by a typical thermal velocity $(k T / m)^{1 / 2}$, and if we estimate the central oneparticle density to be $\rho(0) \sim R^{-3}$ then the prefactor in (38) reduces to $(8 \pi)^{1 / 2} k T i^{2 / 3} /\left(m \gamma R^{2}\right)$, showing clear similarities to the prefactor in equation (43).

\section{Probabilistic approach}

The value of MD simulation is that a wealth of information is available about the system under consideration. We have already exploited this in fixing the parameters for the kinetic and thermodynamic treatments. But we can go further and develop a third, probabilistic approach to estimate the cluster decay rate. It turns out that this approach reproduces the observed decay rates as well as if not better than the approaches described in the previous section, and involves fewer assumptions.

The simulation trajectories are used to calculate the probability density $P(r)=4 \pi r^{2} \rho(r)$ for a particle to be found at a particular distance $r$ from the centre of mass. An example is shown in Figure 9(a). Similarly, the means and variances of the single particle kinetic and potential energies $K$ and $U$, respectively, can be obtained as a function of $r$. The distribution of the potential energy of a particle at a given radius $r$ is approximately Gaussian. The mean potential energy $\bar{U}(r)$ as a function of $r$ is shown in Figure 9(b). Note however that it is the potential of mean force $\Phi(r)$ and not $\bar{U}(r)$ that is responsible for the binding, and one should contrast the shapes of $\bar{U}$ and $\Phi$ in Figure 5

Let us now calculate how often particles violate our cluster definition. First, they must acquire a total energy greater than zero. The probability $P(K>|U| ; r)$ that a particle has $K>|U|$ at a given $r$ can be calculated by combining the kinetic and potential energy probability distributions as shown schematically in Figure 9(c), and integrating over the states whose total energy is positive. For convenience, we make the approximation that the kinetic energy distribution can be treated as a Gaussian. The total energy distribution can then be characterised by adding the means of the individual distributions and their variances in quadrature. This simplification only introduces a small error since it is the larger variance of the potential energy which dominates the combined energy distribution. The resulting probability profile $P(K>|U| ; r)$, is shown in Figure 9(d). As would be expected, if a particle is close to the top of the well then there is a good probability that its total energy will be positive. Conversely, particles very close to the centre of mass will always be energetically bound. The probability density that a particle will be found at position $r$ with positive energy is then obtained by multiplying $P(r)$ by $P(K>|U| ; r)$. The probability $P_{\text {pos }}$ that a particle in the cluster possesses positive energy is then given by integrating the resulting distribution over $r$ as shown in Figure 9(e). The probability that it might then escape is $P_{\text {pos }}\left(1-R_{c}\right)$.

A correction must then be made to account for the fact that the probability density function $P(r)$ acquired from the MD is depleted by the exclusion of configurations corresponding to decay events, since the trajectories are post-processed to remove unbound states as described in reference $\left({ }^{24}\right)$. Therefore, we only see the positive energy excursions that do not lead to decay: in Figure 2 the excursion leading to escape would be ignored and we would count only two events instead of three. The actual number of such events should be obtained from the apparent number by renormalisation by a factor of $1 / R_{c}$, or about $1 / 0.78 \approx 1.28$ for the 50 -cluster. This method also provides the velocity distribution for the escaping atoms at the instant of decay and when they have completely separated from the cluster, as shown in Figure 10.

It remains to determine a timescale $\tau$ for the decay rate, or equivalently an attempt rate for escape $1 / \tau$. We have seen that this timescale is provided in the kinetic approach by the Langevin friction coefficient; in the thermodynamic approach by a capture rate of monomers by a cluster, together with a detailed balance condition, and in other ways in other treatments ${ }^{15}$. We estimate the timescale from our MD simulations in the following way. Encounters with neighbouring particles cause fluctuations in the energy of a particle. These fluctuations drive the slower diffusion of the particle's position within the potential well, but are also the driving force behind the excursion of a particle into a positive energy state. We regard the energy fluctuation timescale as the inverse of the frequency of attempts to achieve positive energy. The timescale $\tau(r)$ is therefore obtained by calculating the average time for the total energy of a particle to pass through its mean value at a given value of $r$, and it is the timescale $\tau_{\text {edge }}$ for $r$ near the edge of the cluster that is relevant to the escape process. The decay rate of a cluster of size $i$ is then given by:

$$
\gamma_{i}^{\mathrm{prob}}=\frac{P_{\mathrm{pos}}\left(1-R_{c}\right)}{\tau_{\text {edge }}(i) R_{c}}
$$

Figure 11 shows the mean lifetimes obtained using this probabilistic scheme for a range of cluster sizes and energies. There is clearly an excellent agreement with the results obtained previously ${ }^{24}$ through direct simulation. The probabilistic, single particle model of the cluster dynamics therefore provides a very good representation of the complex many body problem. 


\section{CONCLUSIONS}

In this paper we have developed a two statistical models, one based on stochastic kinetics and the other on probabilities, to account for the observed rates of decay of simple molecular clusters. The stochastic kinetics approach is based on a representation of single molecule motion using a Langevin equation of motion, and the probabilistic model uses data acquired from molecular dynamics simulations to determine the statistics of how often molecules violate the imposed cluster definition and leave.

These statistical models enable us to view the evaporation of a molecule from a quasi-bound many-body system as a single particle escape problem. Both the kinetic and the probabilistic models can reproduce the measured cluster lifetimes to a high degree of accuracy. This suggests that it should be possible to develop methods to obtain decay rates for clusters that are so long lived that their lifetime would be impossible to measure directly. The accuracy of the methods in predicting cluster lifetimes for such systems will depend upon the amount of data available for use in the analysis. In particular, it is necessary to generate some nearly unbound configurations in order to determine the depths of the potential energy well and the potential of mean force. Simulation data where molecules are extracted slowly from the cluster by an external force, or allowed to drift towards the cluster from outside the simulation cell, could be employed where necessary to provide the required information.

These schemes are very similar in spirit to the potential of mean force calculations that are routinely used in MD studies to obtain thermodynamic quantities for complex systems ${ }^{34}$. Our methods are precisely designed to enable us obtain accurate kinetic data for the study of non-equilibrium processes, such as nucleation.

We have taken the view here that kinetic or probabilistic modelling provides perhaps the most realistic basis for determining the rate of decay, though equilibrium thermodynamic methods have traditionally been the principal tools in this area. However, we have made some progress towards establishing connections between the kinetic and thermodynamic approaches. In particular, we have shown that the depth of the confining potential of mean force, which is a major ingredient in the kinetic statistical decay rate, may be related to the difference in free energy of the clusters with and without the departing molecule, as long as the cluster is to a large degree in internal thermal equilibrium. The kinetic rate of escape is therefore related to equilibrium thermodynamic properties of the cluster. This conclusion has been suggested in the past by identifying nucleation rates derived by kinetic schemes with rates derived on the basis of thermodynamic transition state theory. The uncertainty in the calculated decay rate lies, as has often been found before, in determining the so-called kinetic prefactor, or equivalently a timescale. In our kinetic approach, the timescale is provided by the phenomenological Langevin friction coefficient, while the thermodynamic methods used here appeal to a principle of detailed balance and employ a timescale from the collision rate between monomers and clusters. Other thermodynamic approaches provide timescales in different ways. In the probabilistic approach used here, the timescale is extracted from the fluctuation behaviour of single particle energies. Ultimately, it is a comparison with the decay rates determined by microscopic molecular simulation that test the validity of each of these approaches.

This connection between kinetic and thermodynamic approaches reveals potential points of departure too. The solutions to the Langevin equation we employ are valid in the strong friction regime. How does the kinetic decay rate compare with a free energy difference when particle inertia is taken into account (weak friction)? In this situation, the relatively slow collision rate between particles within the cluster may mean that the full range of microcanonical states is not explored over the timescale of cluster decay. Similarly, systems that are slow to equilibrate after cluster growth may not fully explore conformational space before a subsequent monomer collision occurs, so that the current state of the cluster (and hence its decay rate) becomes coupled to cluster growth. In both of these situations, the relationship between the free energy and the decay rate becomes less obvious. There are clearly several areas where the relationship between the kinetics and thermodynamics of cluster decay is yet to be explored.

\section{Acknowledgments}

This work was supported by the U.K. Engineering and Physical Science Research Council, under grant GR/N23400/01.

1 R. Becker and W. Döring, Ann. Phyz. (Leipzig) 21, 719 (1935).

2 D. Zubarev, V. Morozov, and G. Röpke, Statistical Mechanics of Nonequilibrium Processes Vol. 1: Basic Concepts, Kinetic Theory (Berlin: Akademie, 1996).

3 D. Kashchiev, Nucleation: Basic theory with Applications (Butterworth Heinemann, 2000).

${ }^{4}$ S. Ono and S. Kondo, Handbuch der Physik vol 10, Structure of Liquids (Springer-Verlag, Berlin, 1960), p. 134.

5 I. J. Ford, Phys. Rev. E 56, 5615 (1997).

6 J. K. Lee, J. A. Barker, and F. F. Abraham, J. Chem. Phys. 58, 3166 (1973).

7 B. N. Hale, Australian J. Phys. 49, 425 (1996).

8 I. Kusaka, Z. G. Wang, and J. H. Seinfeld, J. Chem. Phys. 108, 3416 (1998).

9 P. R. ten Wolde and D. Frenkel, J. Chem. Phys. 109, 9901 (1998). 
10 K. J. Oh and X. C. Zeng, J. Chem. Phys. 110, 4471 (1999).

11 S. M. Kathmann, G. K. Schenter, and B. C. Garrett, J. Chem. Phys. 111, 4688 (1999).

12 B. Chen, J. I. Siepmann, K. J. Oh, and M. L. Klein, J. Chem. Phys. 115, 10903 (2001).

13 L. Onsager, Phys. Rev. 37, 405 (1931).

14 D. Reguera, J. M. Rubí, and A. Pérez-Madrid, Physica A 259, 10 (1998).

15 G. K. Schenter, S. Kathmann, and B. Garrett, J. Chem. Phys. 109, 7951 (1999).

16 F. H. Stillinger, J. Chem. Phys. 38, 1486 (1963).

17 J. Xie, J. A. Northby, , and D. L. Freeman, J. Chem. Phys. 95, 3022 (1991).

18 A. A. Lushnikov and M. Kulmala, Phys. Rev. E 58, 3157 (1998).

19 R. Soto and P. Cordero, J. Chem. Phys. 110, 7316 (1999).

20 B. Senger, P. Schaaf, D. S. Corti, R. Bowles, J. C. Voegel, and H. Reiss, J. Chem. Phys. 110, 6421 (1999).

21 R. Bahadur and R. B. McClurg, J. Phys. Chem. 105, 11893 (2001).

22 L. A. Pugnaloni and F. Vericat, J. Chem. Phys. 116, 1097 (2002).

23 J. C. Barrett, J. Chem. Phys. 116, 8856 (2002).

24 S. A. Harris and I. J. Ford, J. Chem. Phys 118, 9216 (2003).

25 G. K. Schenter, S. M. Kathmann, and B. C. Garrett, J. Chem. Phys. 116, 4275 (2002).

26 P. Schaaf, B. Senger, J. C. Voegel, R. K. Bowles, and H. Reiss, J. Chem. Phys. 114, 8091 (2001).

27 T. L. Hill, Statistical Mechanics: principles and selected applications (McGraw-Hill, 1956).

28 B. Nowakowski and E. Ruckenstein, J. Chem. Phys. 94, 1397 (1991).

29 B. Nowakowski and E. Ruckenstein, J. Chem. Phys. 94, 8487 (1991).

${ }^{30}$ H. Risken, The Fokker-Planck equation: methods of solution and applications, 2nd edition (Springer, 1989).

31 P. Hänggi, P. Talkner, and M. Borkovec, Rev. Mod. Phys. 62, 251 (1990).

32 H. A. Kramers, Physica (Utrecht) 7, 284 (1940).

33 D. W. Oxtoby and R. Evans, J. Chem. Phys. 89, 7521 (1988).

34 E. Darve and A. Pohorille, J. Chem. Phys. 115, 9169 (2001). 


\section{Figures}

Figure 1. In the Kramers problem, the escape of a particle is considered for the type of potential shown in (a), with a locally harmonic well centred at $r=0$ and a locally harmonic barrier at $r=r_{e}$. For particle escape from a cluster, the confining potential of mean force is more like (b). The escape radius $r_{e}$ is arbitrary, but can be related to a typical position at which the cluster definition is violated.

Figure 2. The total energy and distance from centre of mass of a particle near the edge of a cluster, in a typical example. The vertical arrows indicate the three energy excursions. Only the last of these events leads to cluster decay.

Figure 3. The average radial discrepancy force per unit mass on a particle in the cluster, plotted against particle radial velocity, obtained by analysing the dynamics on a time interval $\delta t=0.4 \mathrm{ps}$. The dotted line represents a fit to the expected linear behaviour. The slope is the negative of the friction coefficient.

Figure 4. The apparent friction coefficient in the Langevin interpretation of the molecular dynamics, for a range of timesteps $\delta t$ used in the evaluation of the particle acceleration. The expected behaviour at large and small $\delta t$ is seen, and the plateau region centred at about $\delta t=0.3$ ps represents the optimal value of the friction coefficient $\gamma$.

Figure 5. The potential of mean force for a 50 atom argon cluster at $T \approx 50.6 \mathrm{~K}$, obtained from averages of the mean force as a function of radius, derived from MD simulation.

Figure 6 . The one particle density profile $\rho(r)$ for the 50 atom argon cluster at an energy of $-2.98 \mathrm{~kJ} \mathrm{~mol}^{-1}$ per particle $(T \approx 50.6$ $\mathrm{K})$, obtained from MD simulation.

Figure 7. Mean lifetimes of argon clusters of various sizes and at a range of total energies, calculated using the kinetic approach of equation (39) (open circles) and measured directly by MD simulation (filled circles).

Figure 8. Mean lifetimes of argon clusters of various sizes and at a range of total energies, calculated using the thermodynamic approach of equation (38) (open circles) and measured by MD simulation (filled circles).

Figure 9. These plots illustrate the probabilistic method for estimating escape probability $P_{\text {esc }}$. In (a) the one-particle probability density $P(r)$ is shown as a function of distance $r$ from the centre of mass. The data shown is for the 50 particle cluster at an energy of $-2.98 \mathrm{~kJ} \mathrm{~mol}^{-124}$. In (b) we show the mean potential energy well created by this 50 particle cluster. In (c), we give, on the left, the normalised probability distributions for the potential energy $U$ (full line) and the kinetic energy $K$ (dashed line) at $r=12 \AA$. For convenience, the observed Maxwell-Boltzmann kinetic energy distribution is approximated by a Gaussian, as shown. The normalised probability distribution for the total energy, shown on the right, is obtained by combining these two distributions. Integrating this distribution for positive energies gives the probability that $K>|U|$ for this value of $r$. The derived probability that a particle has positive energy at a given position $r$ is shown in (d), again for a 50 particle cluster at an energy of $-2.98 \mathrm{~kJ} \mathrm{~mol}^{-1}$ particle ${ }^{-1}$. The probability density that a particle at position $r$ has positive energy is then the product of the distributions in (a) and (d). Two cases are shown in (e), for 50 particle clusters at temperatures of $T=50.6 \mathrm{~K}$ (full line) and $T=55.4 \mathrm{~K}$ (dashed line). The probability that a particle should acquire positive energy $P_{p o s}$ is then the area under these curves.

Figure 10. The velocity distribution for the escaping atoms at the instant of cluster decay (dashed line) compared with that for particles bound within the cluster (dotted line). The data shown is for a 50 particle cluster at $T \approx 50.6 \mathrm{~K}$. The particles that escape are 'colder' than the average: they have given up kinetic energy in favour of potential energy as the bonds with their neighbours are loosened. The energy distribution of the escaped particles when far from the cluster is shown as a solid line. It clearly does not take the equilibrium shape.

Figure 11. A comparison of the cluster lifetimes calculated using the probabilistic method (open circles) with those measured from the MD simulations (filled circles). 
(a)

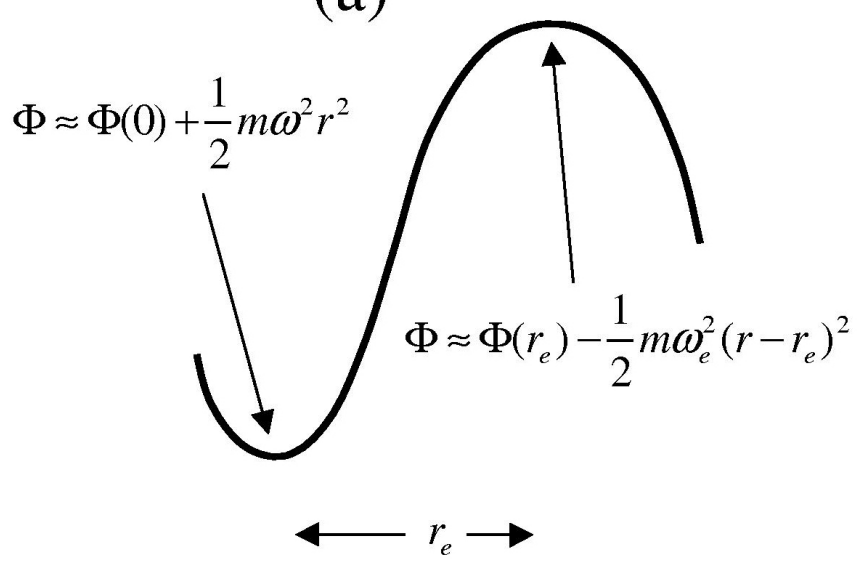

(b)

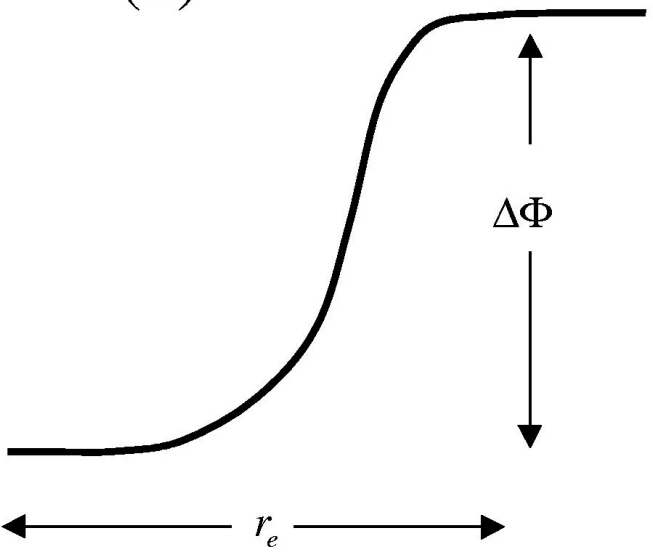

Figure 1: 


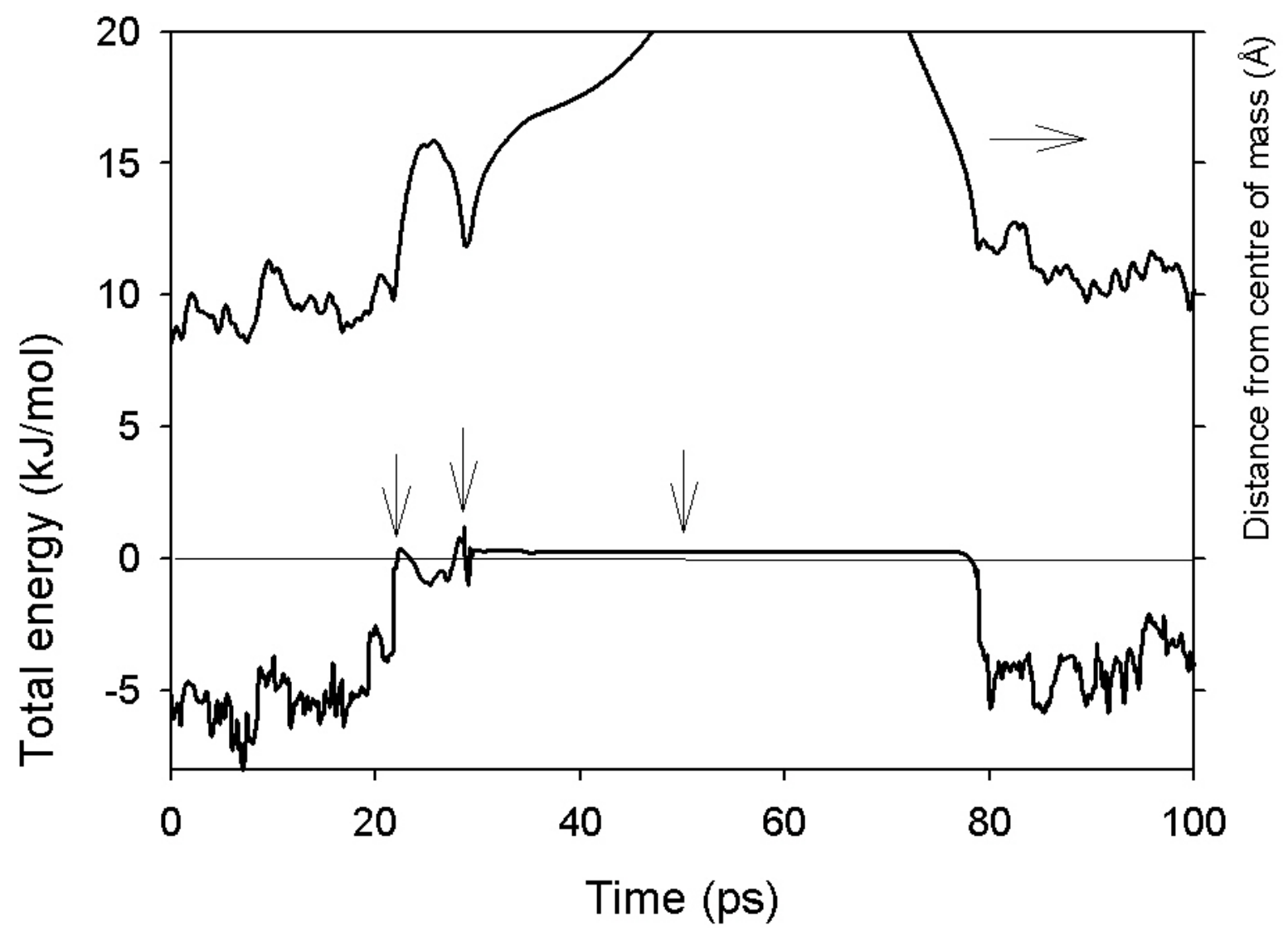

Figure 2: 


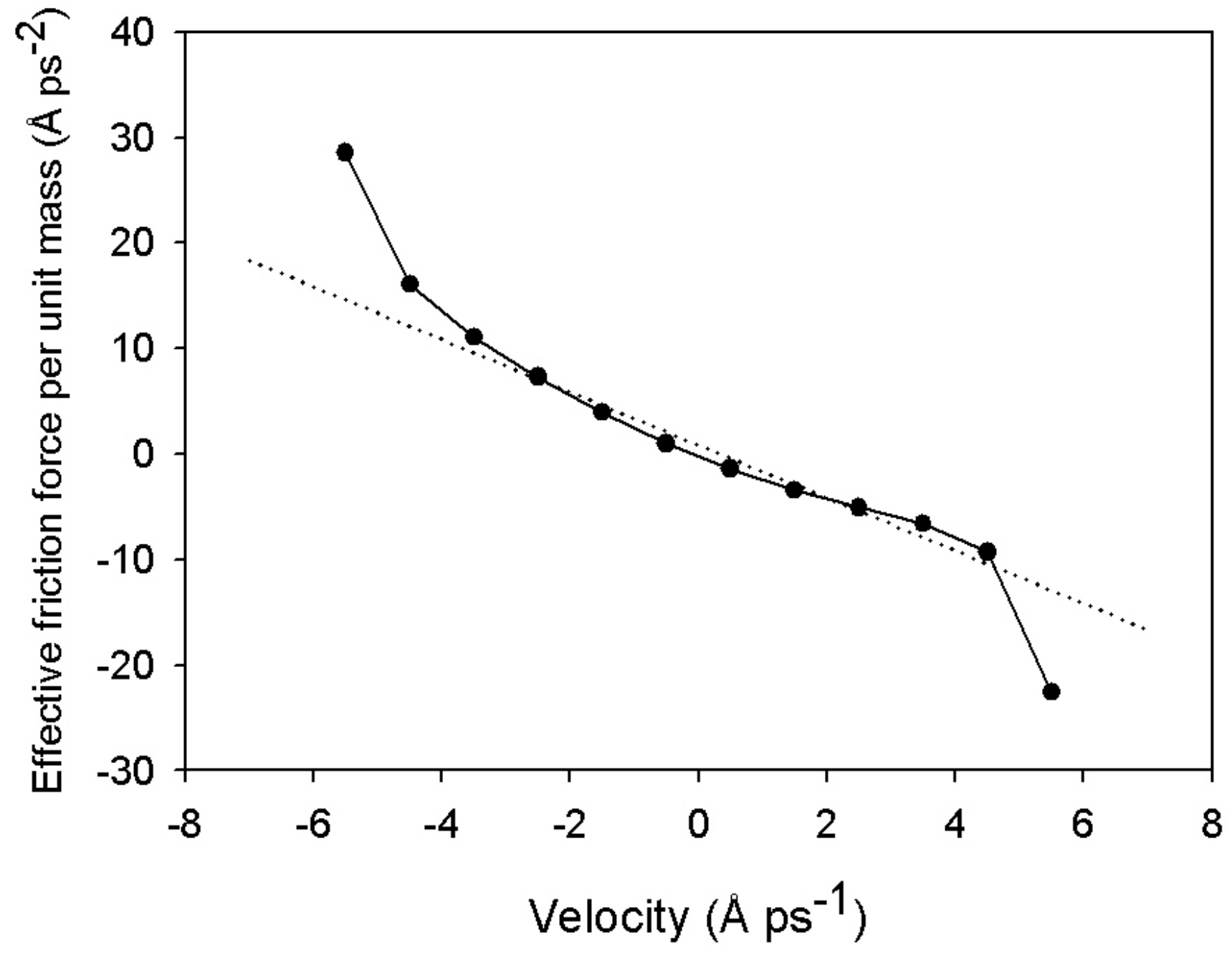

Figure 3: 


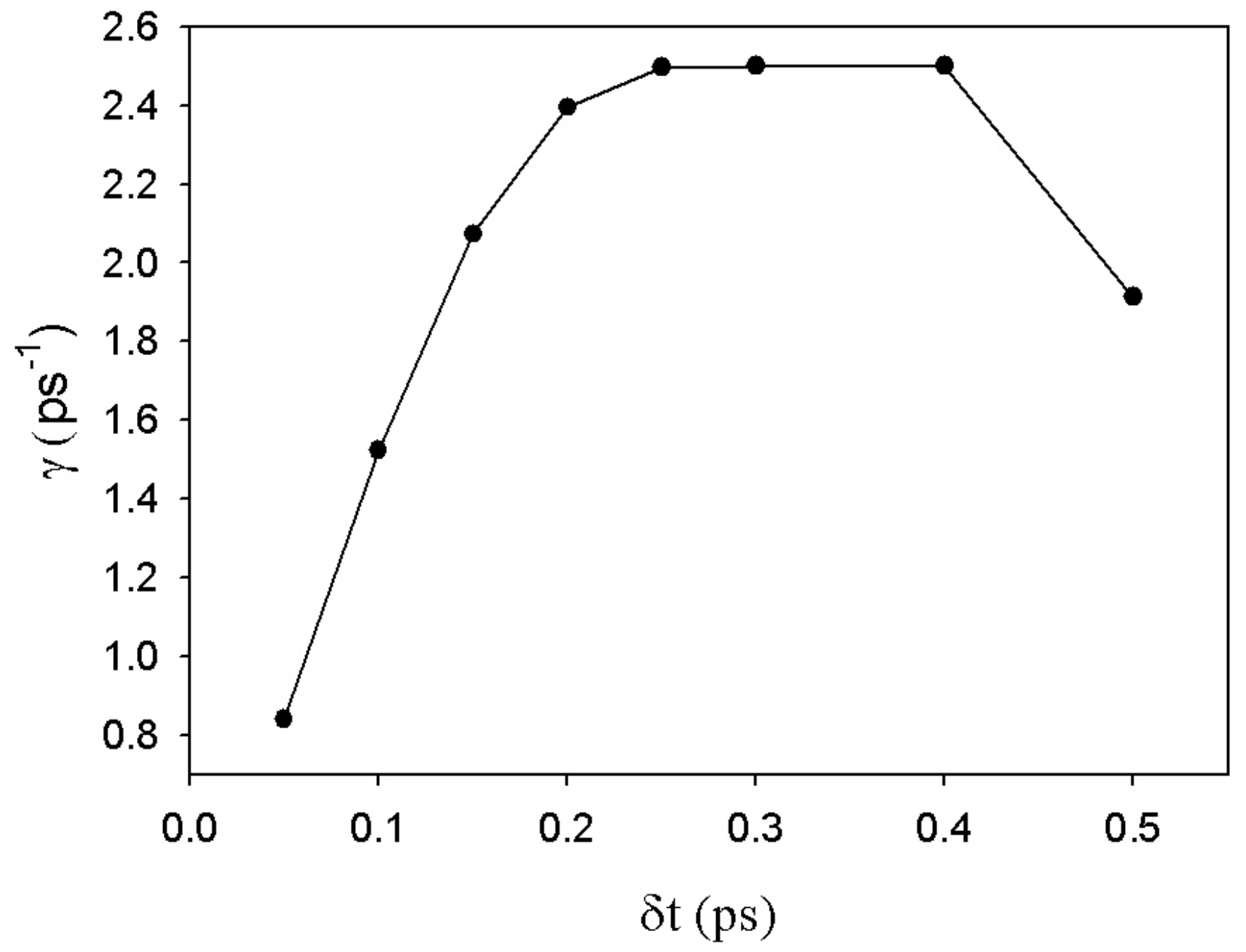

Figure 4: 


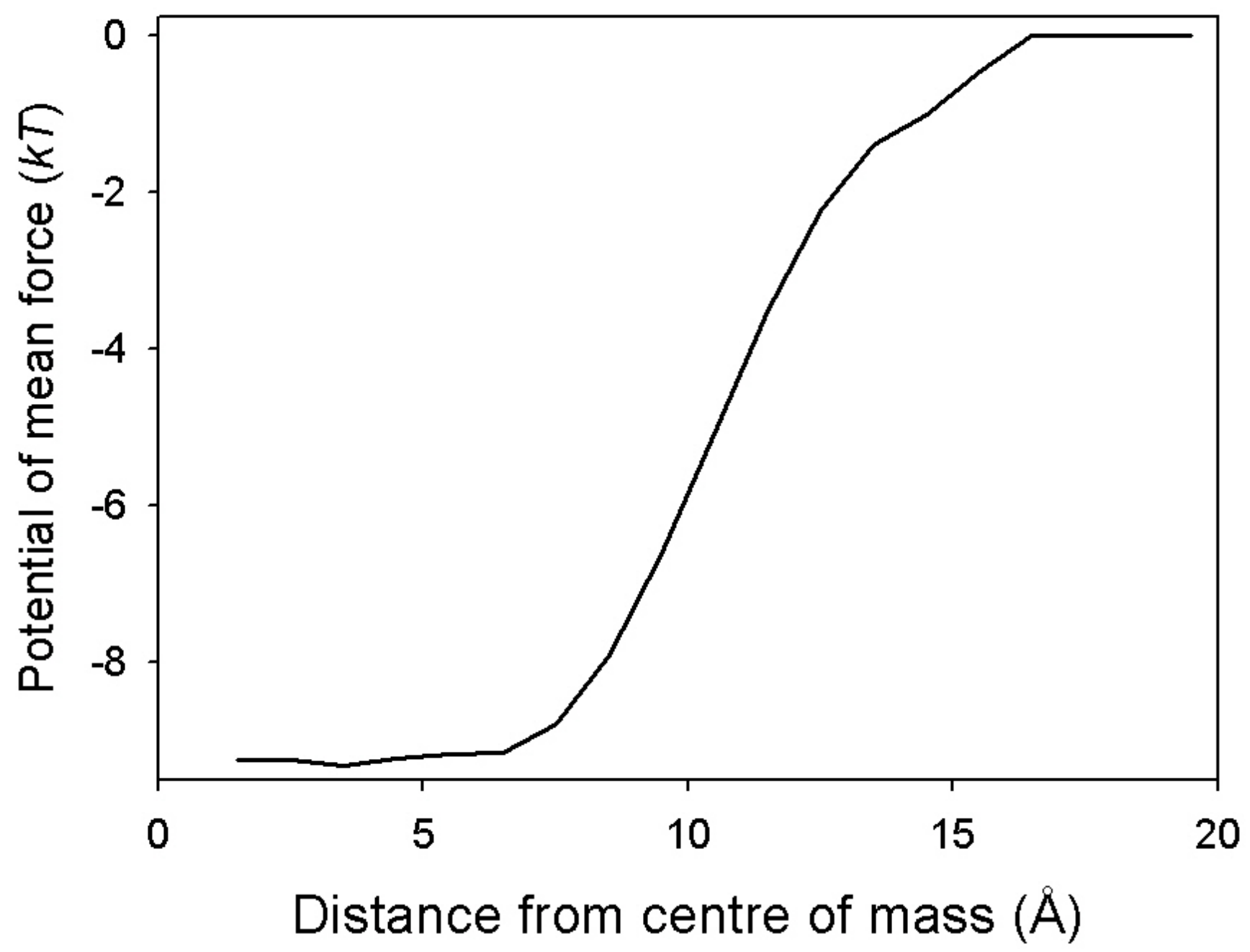

Figure 5: 


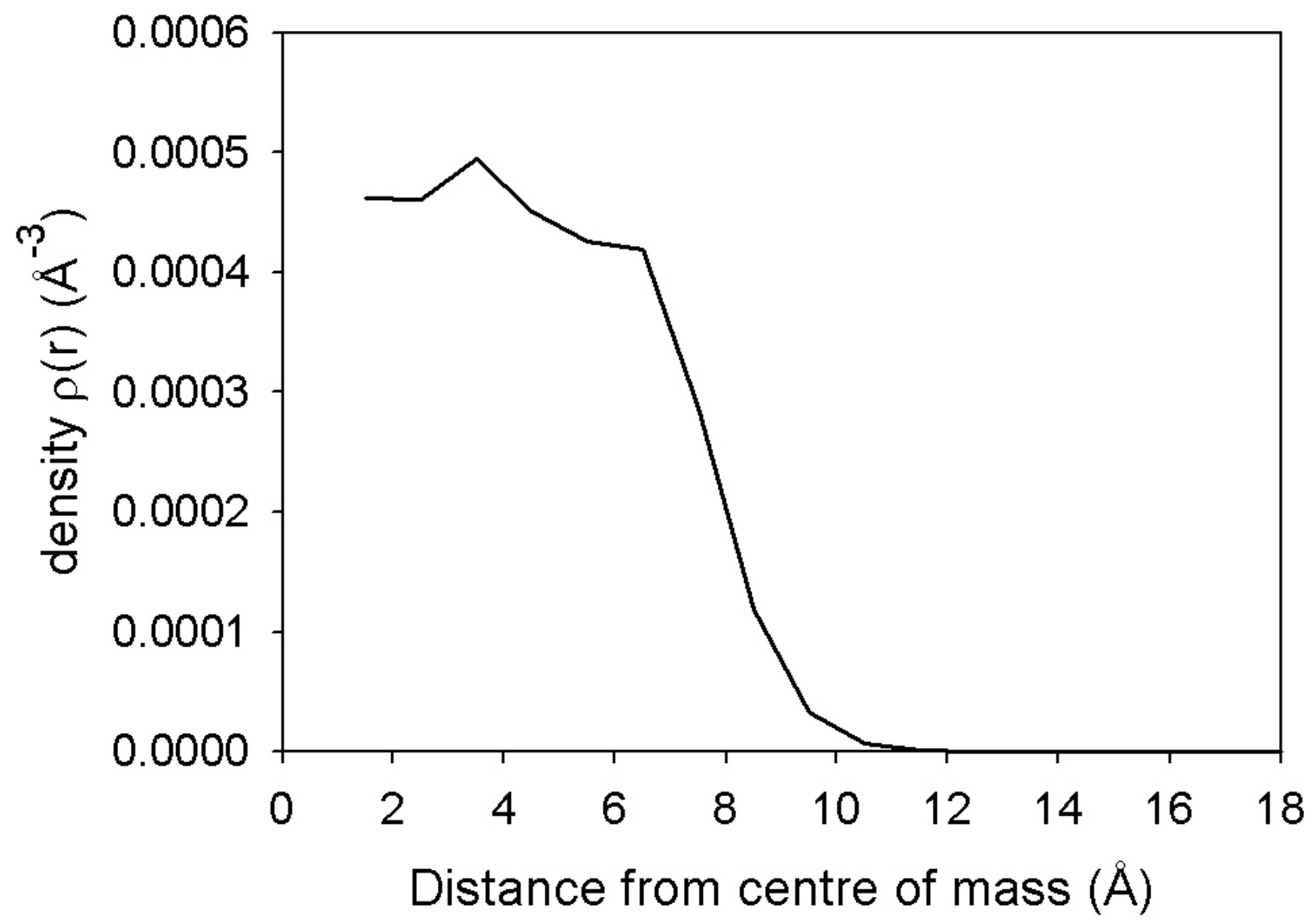




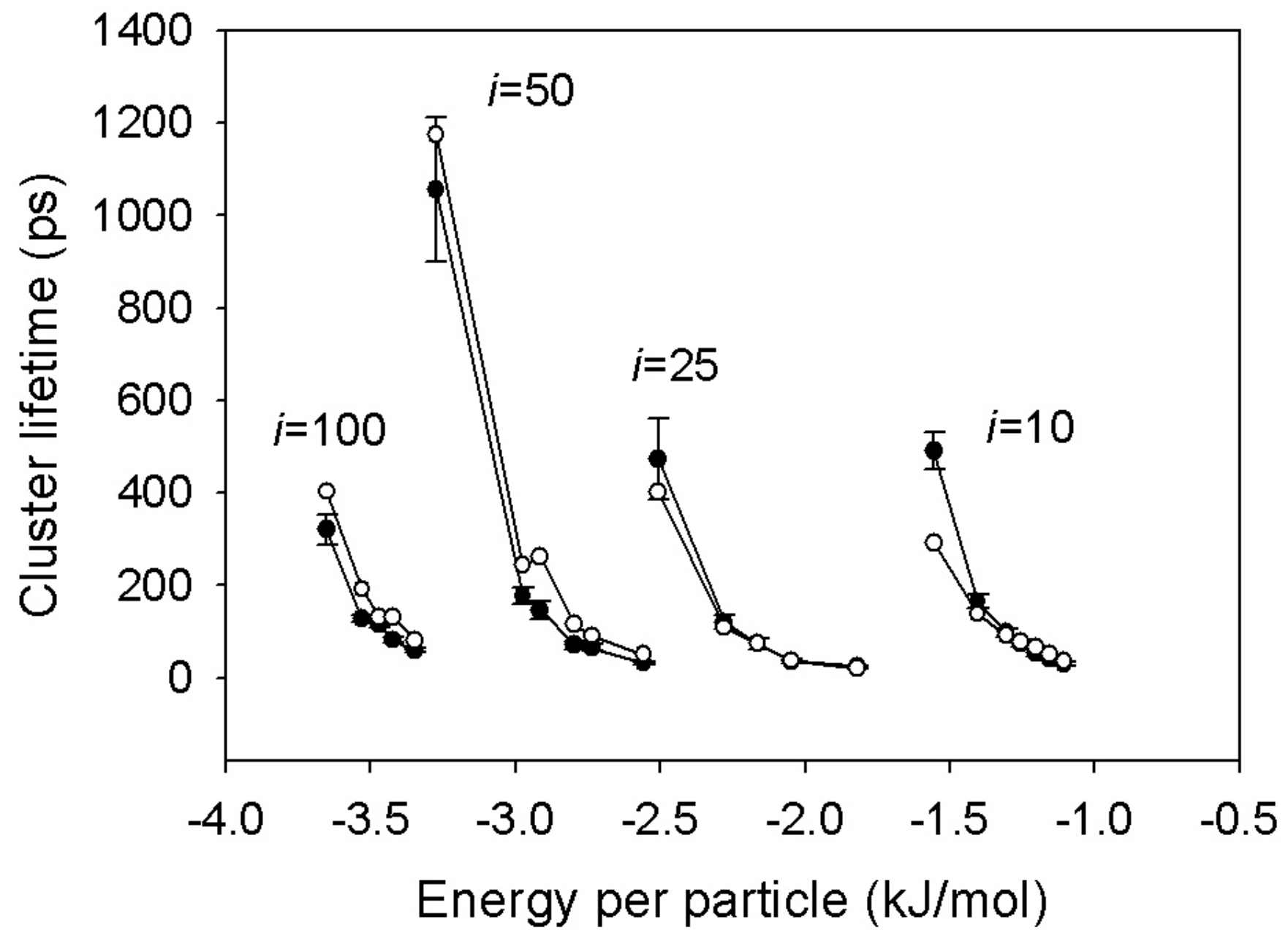

Figure 7: 






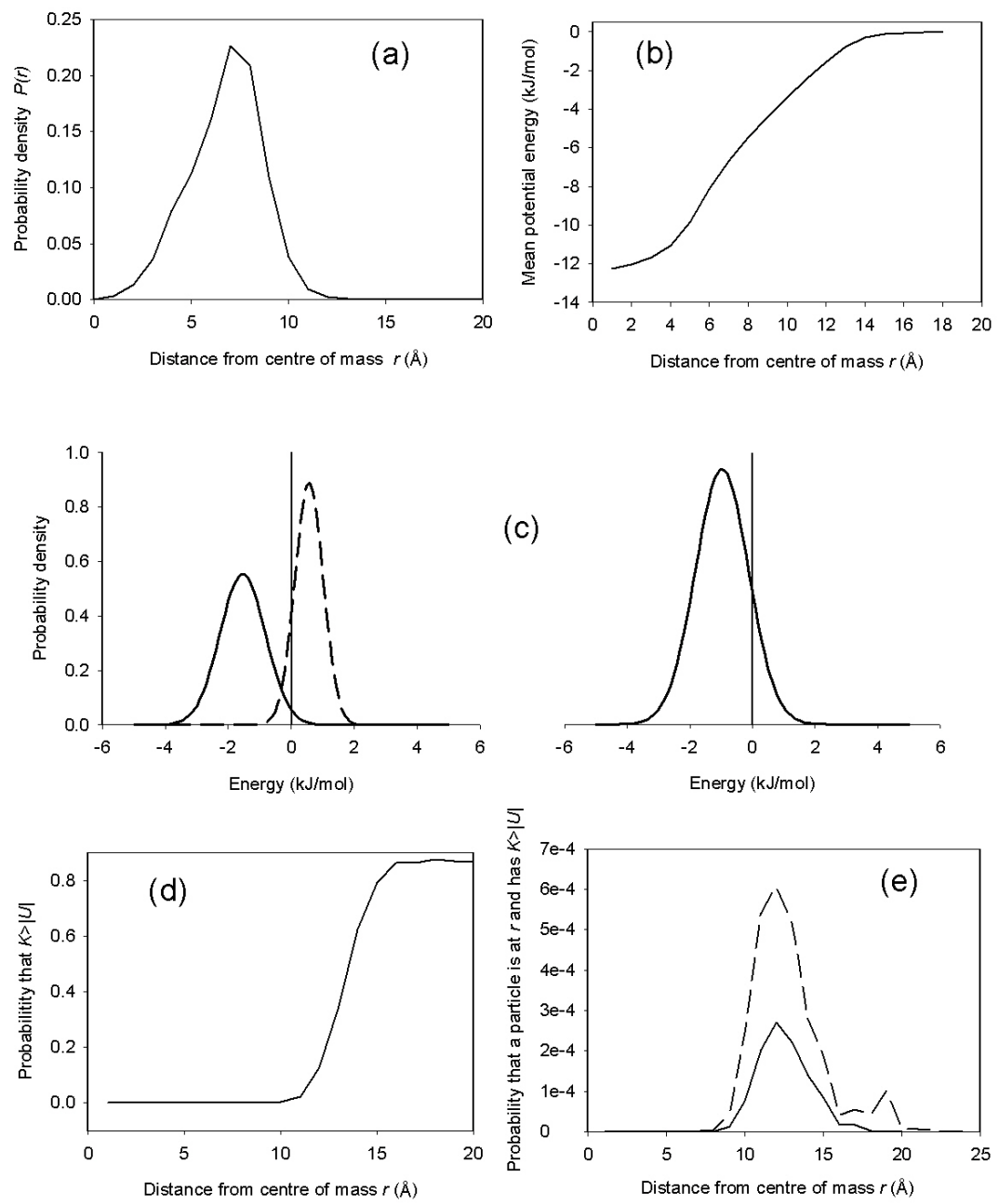

Figure 9: 


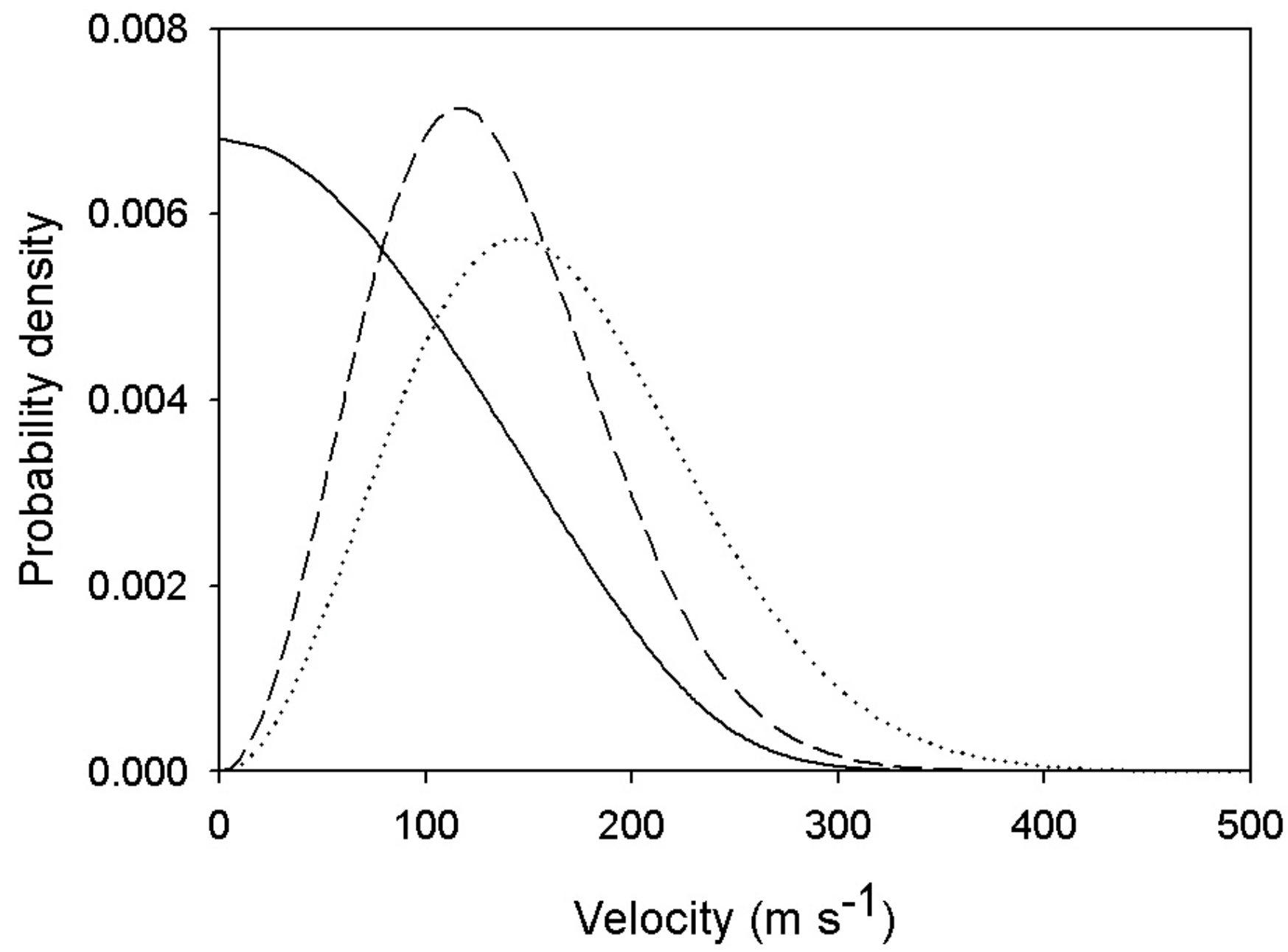

Figure 10: 


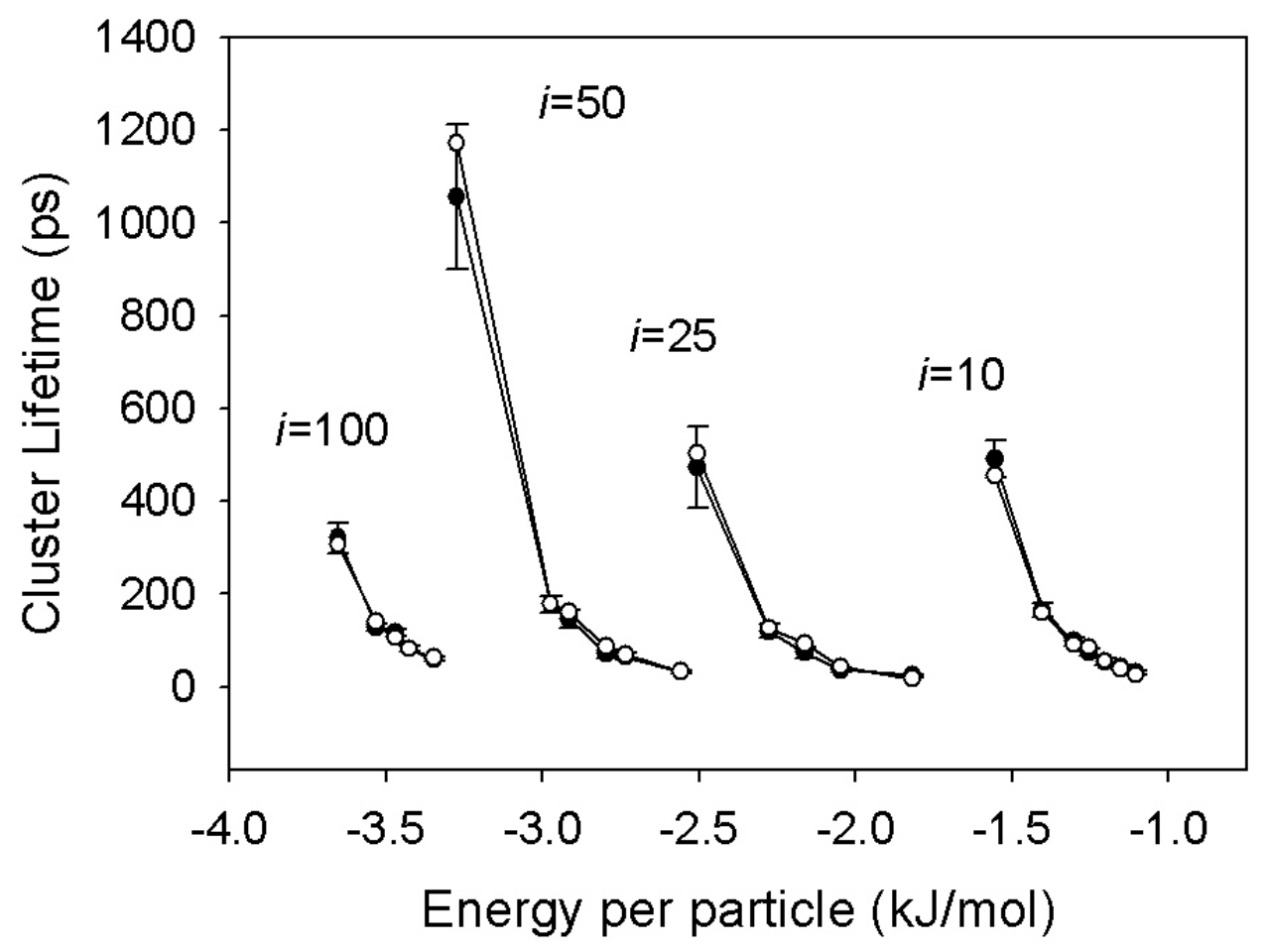

Figure 11: 